RESPONSE

\title{
Response to Letter to Editor (Precision medicine in alcohol dependence: evidence of efficacy and initial support for comparative effectiveness)
}

Neuropsychopharmacology (2018) 43:1801-1802; https://doi.org/ 10.1038/s41386-018-0113-x

We thank Bradford et al. [1] for a thoughtful commentary on our paper about precision medicine in alcohol dependence [2]. As noted by Bradford et al. [1], it is critical to conduct rigorous analyses using pre-registered hypotheses in order to move the field of precision medicine forward. We also contend it is essential to demonstrate that reward/relief drinking moderates the efficacy of medications for alcohol dependence. Demonstrating that naltrexone has a significant effect relative to placebo among reward drinkers provides evidence regarding the efficacy of naltrexone in reward drinkers. Evidence of efficacy can then inform further research efforts that prioritize effectiveness and clinical generalizability, such as direct comparisons of active medications among patient subgroups. With respect to efficacy, Mann et al. [3] hypothesized, a priori, that a subgroup of patients to whom alcohol primarily acts as a positive reinforcer, potentially through increasing available levels of brain endorphins, should respond best to an opioid antagonist (e.g., naltrexone). Conversely, a subgroup of patients to whom alcohol acts as negative reinforcer, potentially because of elevations of brain glutamate levels should respond best to a compound known for its antiglutamatergic action (e.g., acamprosate). Moreover, earlier publications reported drug mechanisms and "putative subgroup dysfunction" [1], such as the role of alcohol-cue-induced brain activation in the ventral striatum as a predictor of naltrexone response [4], as well as the acoustic startle response to distinguish between naltrexone and acamprosate responders [5].

In Mann et al. [2], we examined clinically derived endophenotypes as a basis for differential treatment effects. As hypothesized we found large effect sizes for naltrexone versus placebo in "reward drinkers". We did not find the postulated significant response to acamprosate versus placebo in "relief drinkers", although prior work using the same clinically derived endophenotypes did identify a significant response to acamprosate versus placebo in relief drinkers in the COMBINE study [6]. However, we did not report on the differential efficacy for both drugs in all four subgroups of patients and differences between active medication conditions.

The letter by Bradford et al. [1] gives us an opportunity to add some of those results here. As shown in Fig. 1, among reward drinkers (solid fill), those receiving naltrexone relative to acamprosate had a $45 \%$ decreased likelihood of any heavy drinking, though this effect was non-significant $(p=0.134)$. Among all other drinkers not meeting the reward cut off (diagonal fill), naltrexone was less effective than acamprosate. These reductions in heavy drinking among reward drinkers assigned to naltrexone would most likely be valued among clinicians and patients. The sample of reward drinkers in our study was relatively small ( $n=111$; randomized to either medication or placebo 2:2:1), yet our preliminary findings suggest that a significant naltrexone versus acamprosate effect might be detected in a larger sample of reward drinkers. Future research on clinical generalizability of these findings is an important next step for precision medicine.

Karl Mann (iD) ${ }^{1}$, Corey R Roos ${ }^{2}$ and Katie Witkiewitz (iD ${ }^{2}$ ${ }^{1}$ Department of Addictive Behavior and Addiction Medicine, Central Institute of Mental Health, Medical Faculty Mannheim, Heidelberg University, J5, D-68159 Mannheim, Germany and ${ }^{2}$ Department of Psychology, Center on Alcoholism, Substance Abuse, and Addictions, University of New Mexico, 2650 Yale Blvd SE, Albuquerque, NM 87106, USA

Correspondence: Karl Mann (karl.mann@zi-mannheim.de)

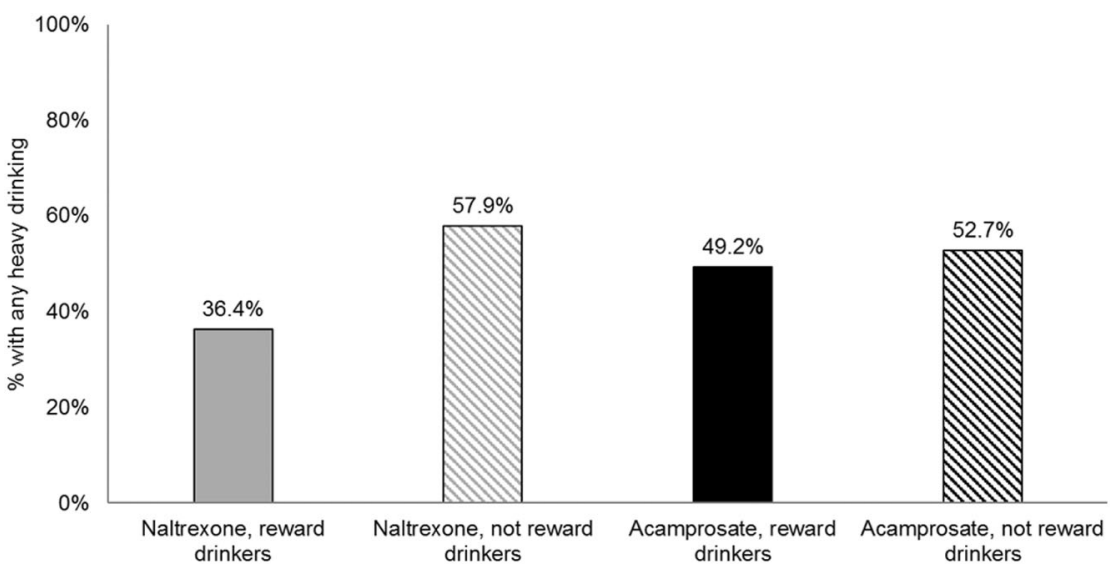

Fig. 1 Comparative effectiveness for reward drinkers versus all other drinkers 


\section{REFERENCES}

1. Bradford DE, Fronk GE, Sant Ana SJ, Magruder KP, Kaye JT, Curtin, JJ (2018). The need for precise answers for the goals of precision medicine in alcohol dependence to succeed. Neuropsychopharmacol. 2018. https://doi.org/10.1038/s41386018-0112-y. Epub 12 June 2018.

2. Mann $K$, Roos CR, Hoffmann S, Nakovics $H$, Leménager $T$, Heinz $A$, et al. Precision medicine in alcohol dependence: A controlled trial testing pharmacotherapy response among reward and relief drinking phenotypes. Neuropsychopharmacol. 2018;43:891-9.

3. Mann K, Kiefer F, Smolka M, Gann H, Wellek S, Heinz A, et al. Searching for responders to acamprosate and naltrexone in alcoholism treatment: Rationale and design of the Predict Study. Alcohol Clin Exp Res. 2009;33:674-83.

4. Mann K, Vollstädt-Klein S, Reinhard I, Leménager T, Fauth-Bühler M, Hermann D, et al. Predicting naltrexone response in alcohol-dependent patients: the contribution of functional magnetic resonance imaging. Alcohol Clin Exp Res. 2014;38:2754-62.

5. Leménager T, Hill H, Reinhard I, Hoffman S, Zimmerman S, Hermann D, et al. Association between alcohol-cue modulated startle reactions and drinking behaviour in alcohol dependent patients-results of the PREDICT study. Int J Psychophysiol. 2014;94:263-71.

6. Roos CR, Mann K, Witkiewitz K. Reward and relief dimensions of temptation to drink: construct validity and role in predicting differential benefit from acamprosate and naltrexone. Addict Biol. 2017;22:1528-39. 